\title{
Prevalence and Correlates of Depressive Symptoms in a Community Sample of People Suffering from Heart Failure
}

\author{
Carolyn L. Turvey, PhD, * Kara Schultz, BA, Stephan Arndt, PhD, ${ }^{*+}$ Robert B. Wallace, MD, $\neq$ \\ and Regula Herzog, PhD"l
}

OBJECTIVES: To examine the rates and correlates of depressive symptoms and syndromal depression in people with self-reported heart failure participating in a community study of people aged 70 and older.

DESIGN: Cross-sectional.

SETTING: Community-based epidemiological study of older people from the continental United States.

PARTICIPANTS: Six thousand one hundred twenty-five older people participating in the longitudinal study of Assets and Health Dynamics. Participants had to be born in 1923 or earlier.

MEASUREMENTS: The short-form Composite International Diagnostic Interview assessed syndromal depression, and a revised version of the Center for Epidemiologic Studies-Depression scale assessed depressive symptoms. Medical illness was based on self-report. The authors compared the rates of syndromal depression and individual depressive symptoms in people with self-reported heart failure $(\mathrm{n}=199)$ with those in people with other heart conditions $(\mathrm{n}=1,856)$ and with no heart conditions $(\mathrm{n}=$ 4,070).

RESULTS: Eleven percent of those with heart failure met criteria for syndromal depression, compared with $4.8 \%$ of people with other heart conditions and $3.2 \%$ of those with no heart conditions. The association between heart failure and depression held even after controlling for disability,

From the *Department of Psychiatry, College of Medicine, ${ }^{\dagger}$ Mental Health Clinical Research Center, Department of Psychiatry, ${ }^{ \pm}$Department of Preventive Medicine, School of Public Health, ${ }^{\circledR}$ College of Medicine, University of Iowa, Iowa City; and "Institute for Social Research, Institute of Gerontology, Department of Psychology, University of Michigan, Ann Arbor, Michigan.

This research was supported by the National Institutes of Health Grant 1 U01 AG12980 as part of the project entitled "The Oldest Old Supplement to the Health and Retirement Survey" and 1-2115200 the "Health and Retirement Survey" (Drs. Wallace and Herzog), National Institute of Mental Health Grants \#P50 MH43271, MH40856, and MH31593 (Dr. Arndt) and K01-MHO1964-01 (Dr. Turvey).

Parts of this paper were presented at the annual meting for the American College of Neuropsychopharmacology, Acapulco, Mexico, 1999.

Address correspondence to Carolyn L. Turvey, PhD, Psychiatry ResearchMEB, University of Iowa, Iowa City, IA 52242. E-mail: carolyn-turvey @uiowa.edu reported fatigue and breathlessness, and number of comorbid chronic illnesses.

CONCLUSION: Community-living older people with self-reported heart failure were at approximately twice the risk for syndromal depression of the rest of the community. Although fatigue and functional disability were also related to depression in this sample, these variables did not account for the association between syndromal depression and self-reported heart failure. J Am Geriatr Soc 50:20032008, 2002.

Key words: depression; community-living older people; heart failure

$\mathrm{H}$ eart failure is one of the leading causes of morbidity and mortality in older Americans. ${ }^{1}$ The incidence and prevalence of heart failure increases with age, ${ }^{2}$ which means that the aging of the U.S. population will make this an even larger public health problem. Heart failure patients suffer significant depressive morbidity that is best characterized when minor depression and adjustment disorder and major depression are assessed. ${ }^{3-10}$ Depressive symptoms predict greater mortality in heart failure patients, ${ }^{11}$ so research investigating depression may provide information on whether treated depression will improve not only the quality of life for these patients, but also the course of the medical illness.

Koenig ${ }^{6}$ reported that $36.5 \%$ of 107 hospitalized heart failure patients met criteria for major depression and $21.5 \%$ for minor depression. Freedland et al. ${ }^{5}$ also working with inpatients, found a lower rate of $17 \%$, comparable with the $15 \%$ reported by Maricle et al., ${ }^{7}$ for major depression in a group of patients referred for heart transplant. The Maricle et al. study also found that 34\% met criteria for adjustment disorder with depressed mood and 9\% met criteria for dysthymia. In addition to Diagnostic and Statistical Manual of Mental Disorders (DSM)-based studies, four studies examining quality of life have found elevated rates of distress, hopelessness, and mood disruption in heart failure patients. ${ }^{4,9,10,12}$ 
Most studies of heart failure have been based on patient samples with severe heart failure. Such patient samples tend to have higher rates of comorbidity with depression and with other medical illnesses associated with depression such as stroke. ${ }^{13}$ Most prior studies have used inclusive diagnostic approaches where the source of symptoms that could be attributed to medical illness or depression was not determined and the presence of such symptoms, such as low energy or difficulty eating, were counted toward a diagnosis of depression.

There is considerable empirical support for an inclusive approach to the diagnosis of depression in primary care patients. ${ }^{14,15}$ However, patients with heart failure, particularly those in the hospital, may report low energy, difficulty enjoying themselves, and poor sleep due to apnea. These symptoms do not necessarily reflect depression but may account for the higher rates of depression reported in patients with heart failure. More research is needed to separate the relative contribution of illness severity to the elevated rates of depression in heart failure patients so that the appropriate patients are diagnosed with depression and receive adequate treatment.

This study presents the rates of syndromal depression in people with heart failure participating in a community study of people aged 70 and older and their spouses. To complement studies that use patient samples only, a community sample was used to compare syndromal depression in people with heart failure with people with other heart conditions and those with no heart conditions. The large sample size allowed for a multivariate analysis that provided estimates of depression in heart failure patients while controlling for indications of medical severity such as number of comorbid illnesses, presence of persistent fatigue or apnea, and functional disability.

\section{METHODS}

\section{Sample}

The Assets and Health Dynamics (AHEAD) study ${ }^{16}$ is a companion study to the Health and Retirement Survey ${ }^{17}$ and is intended to investigate the effect of health transitions on personal financial management, service and public program use, and intergenerational transfer of assets. The two sampling frames for the study were the 1992 screening of housing units enumerated for the Health and Retirement Survey and the Health Care Finance Administration's (now called Center for Medicare and Medicaid Services) Master Enrollment file of Medicare enrollees who were living in a household. Primary respondents had to be aged 70 and older; if respondents were married, their partners participated even if their partner was younger than 70 . Although the initial sampling frame excluded institutionalized older people, respondents who were institutionalized after Wave 1 remained in the study and were interviewed at Wave 2. This is a cross-sectional study with the exception that the Wave 1 Center for Epidemiologic Studies-Depression Scale (CES-D) score was collected at Wave 1. All other variables presented were collected at Wave 2 at the same time that the depression measures were administered.

The first wave of AHEAD occurred in 1993/94, the second wave in 1995/96. Wave 1 included 8,406 respondents. Of these, 828 died between Wave 1 and Wave 2. Of the 7,578 living at Wave 2, 7,038 completed the second interview, yielding a $93 \%$ follow-up rate. Depression measures were not administered to respondents receiving proxy interviews at Wave $2(\mathrm{n}=901)$, and 12 subjects did not provide adequate information. The final study sample size was 6,125 . The study group was $38 \%$ male and $86 \%$ Caucasian, 12\% African American, and 2\% Native American, Asian, or Pacific Islander. The mean age \pm standard deviation was $76 \pm 6$. Sixty-one percent of the participants had at least a high-school education. All respondents provided verbal informed consent, and internal ethics review board approval was obtained. Respondents aged 70 to 79 were interviewed by telephone, and respondents aged 80 and older were interviewed in person.

\section{Syndromal Depression and Depressive Symptoms}

Syndromal depression was assessed using a short form of the Composite International Diagnostic Interview (CIDI), a structured interview originally developed by the World Health Organization that was subsequently adapted for the National Comorbidity Study. ${ }^{18}$ This measure was collected only in the second wave of the AHEAD study, so longitudinal data on this measure are not available. The short-form CIDI assesses 1-year prevalence for eight of the nine possible associated symptoms required for diagnosis of a depressive episode by both DSM-III-R and DSM-IV criteria. The only symptom excluded was motor slowing or agitation.

The short-form CIDI does not exclude the diagnosis in the context of bereavement or organic causes. The threshold of five symptoms or more, including at least depressed mood or anhedonia and a 2-week duration was required for diagnosis. In a prior validation study, a five-symptom threshold on the short-form CIDI corresponded to a positive predictive value of 0.89 for diagnosis on the full-scale CIDI (Kessler $\mathrm{R}$ et al., unpublished data) using respondents from the National Comorbidity Survey. A four-item scale of nonsomatic depressive symptoms was constructed from the short-form CIDI. It included depressed mood, poor concentration, feelings of worthlessness, and thoughts of death.

The revised CES-D was administered in both Wave 1 and Wave 2 of AHEAD to all nonproxy interviews. The revised CES-D is an eight-item version of the original scale $^{19}$ with a yes/no response format for each question. Subjects were asked to endorse the item if they experienced the symptom "much of the time during the past week." Given the association between heart conditions, depression, and mortality, one might expect Wave 1 patients with heart failure and elevated depressive symptoms to die more quickly, thereby leading to an underestimate of the association in the Wave 2 sample, but, for people reporting heart failure at Wave 2, the mean revised CES-D score at Wave 2 (mean $=2.54$ ) was actually higher than at Wave 1 (mean $=2.25)$.

\section{Health Measures}

Health measures were based on self-report. Participants reported whether a medical doctor had diagnosed them with cancer, diabetes mellitus, high blood pressure, lung disease, stroke, or arthritis. Participants were asked whether a doctor had told them that they had had a heart 
attack or had coronary heart disease, angina pectoris, heart failure, or other heart problems. Presence or absence of heart failure was assessed only at Wave 2. In addition, all study participants were asked whether they had persistent or troublesome shortness of breath while awake and persistent or troublesome fatigue or exhaustion. Three groups were formed based on heart condition status: heart failure $(\mathrm{n}=199)$, other heart condition $(\mathrm{n}=1,856)$, and no heart conditions $(n=4,070)$. People in the heart failure group were asked whether they had been hospitalized for heart failure within the last 2 years and whether they were taking medication for their condition.

\section{Cognitive Measures}

The development of the AHEAD cognitive measures was adapted from the Telephone Interview for Cognitive Status, ${ }^{20}$ which was modeled after the Mini-Mental State Examination $^{21}$ to be administered over the telephone. A total score was determined by summing the serial 7 , immediate and delayed free-recall, and mental state scale totals.

\section{Physical Function Impairment}

Impairment in physical functioning was assessed using 16 items reflecting standard activities of daily living (ADLs) plus mobility and strength. We calculated the proportion of the sample reporting any difficulty for each item and then ranked the items based on the resulting percentages. The items constituting each domain and the percentage of the total sample who endorsed each item were as follows.

1. ADLs: eating $(3.6 \%)$, using the toilet $(7.2 \%)$, getting out of bed $(7.7 \%)$, walking across the room $(9.1 \%)$, bathing $(9.8 \%)$, and dressing $(12.5 \%)$

2. Activities of moderate mobility and strength: sitting for about 2 hours $(17.7 \%)$, reaching arms above shoulder level $(18.6 \%)$, walking one block $(18.9 \%)$, walking up one flight of stairs without resting $(27.3 \%)$, and lifting or carrying weights over 10 pounds $(35 \%)$

3. Activities of greater mobility or strength: walking several blocks without resting (39.5\%); pulling or pushing large objects like a living room chair $(40 \%)$; rising from a chair after sitting for long periods $(41 \%)$; stooping, kneeling, or crouching (49\%); walking several flights of stairs without resting $(60 \%)$

Comparable with a measure recommended by Guralnik et al., ${ }^{22}$ study participants were then grouped in mutually exclusive categories using a four-level variable indicating severity of functional impairment, with 0 indicating no impairment $(\mathrm{n}=1,167 ; 19 \%), 1$ indicating impairment in activities of greater mobility and strength only $(\mathrm{n}=1,502$; $25 \%), 2$ indicating impairment in activities of moderate mobility and strength with no reported impairment in ADLs $(\mathrm{n}=1,985 ; 32 \%)$, and 3 indicating any impairment in basic ADLs ( $\mathrm{n}=1,462 ; 24 \%$ ).

Assessment of instrumental activities of daily living (IADLs) included tasks that require both a cognitive and physical component and therefore constitute a separate indication of disability: meal preparation $(14 \%)$, grocery shopping $(17 \%)$, telephone use $(5 \%)$, taking medication $(9 \%)$, and managing money $(10 \%)$. People who reported any difficult on any of these items were coded as impaired in IADLs $(\mathrm{n}=1,960 ; 32 \%)$.

\section{Statistical Analysis}

Differences between groups on categorical variables were conducted using chi-square test statistic. Fisher exact calculations were performed when expected cell counts were less than five. Comparisons between groups for ordinal variables were tested using the Wilcoxon rank-sum test with a chi-square approximation or a $t$ test when assumptions of normal distribution were met. Logistic regression analysis was used for the multivariate analyses and to obtain odds ratios and $95 \%$ confidence intervals for univariate and multivariate analyses. All tests were two-tailed. To correct for multiple comparisons, only $P$-values $<.01$ were considered significant.

\section{RESULTS}

\section{Comparisons of Syndromal Depression using the Complete Sample}

Eleven percent of those with heart failure met criteria for syndromal depression, whereas $4.8 \%$ of patients with other heart conditions and $3.2 \%$ of those with no heart condition were depressed. People with heart failure had significantly higher rates of syndromal depression on the short-form CIDI than those in the no heart condition group $\left(\chi^{2}=34.6, d f=1, P<.0001\right)$ or the other heart condition group $\left(\chi^{2}=13.4, d f=1, P<.0002\right)$.

A multivariate analysis was conducted using the entire sample except for those with no score on the Wave 1 revised CES-D (Table 1). The outcome was presence or absence of syndromal depression, and the predictor variables were age, sex, years of education, total number of chronic illnesses other than heart conditions, heart condition status (heart failure vs other heart conditions or no heart condition), report of persistent or troublesome shortness of breath while awake, report of persistent or troublesome fatigue or exhaustion, Wave 1 revised CES-D total score, total impairment score for cognition, the physical function disability scale, and impairment in IADLs. Wave 1 revised CES-D score was clearly the strongest correlate of syndromal depression at Wave 2, with greater fatigue and younger age also increasing the odds of depression. Impairment in physical function also predicted depression, with each increment in severity of disability corresponding to approximately $40 \%$ increased odds for depression. Presence of heart failure, when compared with all others, almost doubled the risk of syndromal depression. Total number of chronic illnesses, cognitive impairment, and impairment in IADLs did not predict syndromal depression.

\section{Comparisons of Depressive Symptoms and Nonsomatic Symptoms of Depression}

People with heart failure had higher depressive symptom scores on the revised CES-D than those in the other heart condition group (other heart conditions mean \pm standard deviation $=1.8 \pm 2.1$; heart failure mean $=2.5 \pm 2.2$; t-equal variance $=-4.7, d f=2,053, P<.0001)$ and the no heart condition group (no heart condition mean $=1.3 \pm$ 1.8 ; $\mathrm{t}$-unequal variance $=-8.0, d f=211, P<.0001)$. Using the scale consisting of only the nonsomatic symptoms of depression administered during the short-form CIDI, the heart failure group had more total symptoms than the other heart condition group (other heart condition mean $=$ 
Table 1. Association Between Syndromal Depression on the Short-Form Composite International Diagnostic Interview and Demographic and Clinical Variables: Results from Multiple Logistic Regression $(\mathrm{N}=5,946)$

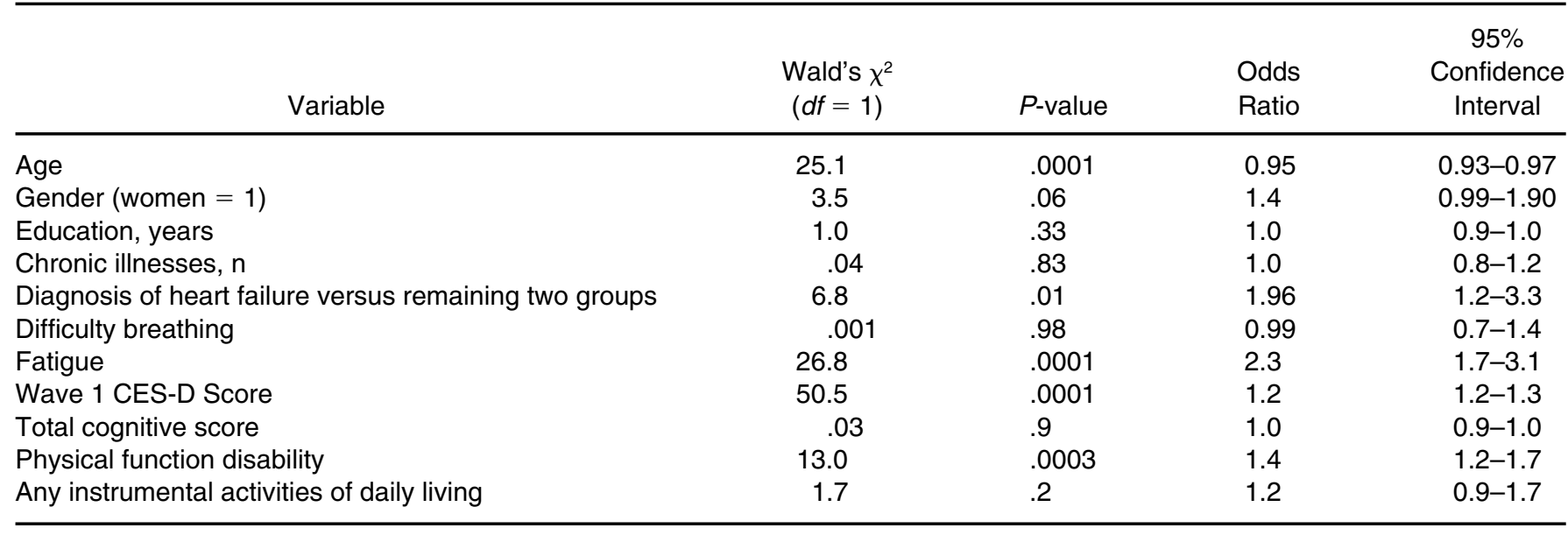

Note: Likelihood ratio test for entire model: $\chi^{2}=244.3, d f=11, P<.0001$. Sample size is somewhat reduced because of missing data on some variables, particularly those who did not complete the revised Center for Epidemiologic Studies-Depression Scale (CES-D) at Wave 1 ( $\mathrm{n}=167$ ) because of proxy status.

$0.2 \pm 0.7$; heart failure mean $=0.45 \pm 1.1 ; \mathrm{t}$-unequal variance $=-3.2, d f=217, P<.002)$ and the no heart condition group (no heart condition mean $=0.12 \pm 0.6$; t-unequal variance $=-4.1, d f=203, P<.0001)$.

A multiple regression analysis was conducted, with the total score on the revised Wave 2 CES-D, a diagnosis of heart failure versus all other heart conditions, and the same covariates used in the logistic regression with syndromal depression. In this analysis, the covariates, particularly Wave 1 CES-D score, comorbid illness, physical and cognitive disability and fatigue (Table 2), entirely accounted for the association between heart failure and depression. An additional multiple regression excluding indicators of breathlessness or fatigue from this model showed that the association between heart failure and depressive symptoms remained (heart failure vs all other $\mathrm{t}=3.06, P<$
.002 ; remaining data from this analysis available on request.)

Analyses of Syndromal Depression with Only Those Who Suffered Heart Failure $(\mathrm{N}=199)$

Comparisons between depressed and nondepressed heart failure patients show no differences in marital status, age, sex, and race (Table 3). There was a small difference between the two groups in percentage with a high school degree, although the difference did not reach the predetermined level of significance. In addition, there were no differences between depressed and nondepressed heart failure patients on indicators of severity such as recent hospitalization, treatment with medication, or self-report of stroke.

Wave 1 depressive symptoms on the revised CES-D were higher in the depressed heart failure group. De-

Table 2. Association Between Depressive Symptoms Measured with the Revised Center for Epidemiologic StudiesDepression Scale (CES-D) and Demographic and Clinical Variables: Results from Multiple Regression $(\mathrm{N}=5,946)$

\begin{tabular}{|c|c|c|c|c|}
\hline Variable & Estimate & Standard Error & $t$ value & $\operatorname{Pr}>t^{*}$ \\
\hline Intercept & 0.72 & 0.30 & 2.4 & 0.02 \\
\hline Age & -0.002 & 0.003 & -0.74 & 0.46 \\
\hline Education, years & -0.01 & 0.006 & -1.5 & 0.14 \\
\hline Chronic illnesses, $\mathrm{n}$ & 0.10 & 0.02 & 3.9 & 0.0001 \\
\hline Diagnosis of heart failure versus remaining two groups & 0.17 & 0.11 & 1.5 & 0.13 \\
\hline Fatigue & 0.92 & 0.07 & 16.1 & 0.0001 \\
\hline Wave 1 CES-D Score & 0.40 & 0.01 & 34.7 & 0.0001 \\
\hline Total cognitive score & -0.02 & 0.004 & -5.6 & 0.0001 \\
\hline Physical function impairment & 0.20 & 0.02 & 8.7 & 0.0001 \\
\hline Any instrumental activities of daily living & 0.19 & 0.05 & 4.2 & 0.0001 \\
\hline
\end{tabular}

Note: For entire model, $\mathrm{F}_{11,5,934}=328.16, P<.0001$. Sample size is somewhat reduced because of missing data on some variables, particularly those who did not com plete the revised CES-D at Wave $1(\mathrm{n}=167)$ because of proxy status.

${ }^{*}$ Probability of obtaining a $t$ value greater than the one obtained from this multiple regression. 
Table 3. Correlates of Syndromal Depression in Heart Failure Patients: Univariate Analyses

\begin{tabular}{|c|c|c|c|c|c|}
\hline Variable & $\begin{array}{l}\text { Nondepressed } \\
\quad(n=177)\end{array}$ & $\begin{array}{l}\text { Depressed } \\
(\mathrm{n}=22)\end{array}$ & $\begin{array}{l}\chi^{2}, t \text { value, or } \\
\text { Fisher Exact }\end{array}$ & $d f$ & $P$-value \\
\hline \multicolumn{6}{|l|}{ Demographics } \\
\hline Age, mean $\pm S D$ & $77 \pm 6.4$ & $75 \pm 6.1$ & 1.28 & 196 & 2 \\
\hline Female, \% & 62 & 64 & 0.02 & 1 & .9 \\
\hline Race, $\%$ & & & 0.8 & 2 & .7 \\
\hline White & 92 & 95 & & & \\
\hline African American & 5 & 5 & & & \\
\hline Asian, Native American, or Pacific Islander & 3 & 0 & & & \\
\hline Marital status, Wave $2, \%^{*}$ & & & & & .9 \\
\hline Divorced & 5 & 4 & & & \\
\hline Widowed & 45 & 41 & & & \\
\hline Married or living with someone & 49 & 55 & & & \\
\hline Never married & 2 & 0 & & & \\
\hline Education, years, mean \pm SD & $10.5 \pm 3.5$ & $8.8 \pm 4.7$ & 4.1 & 1 & .04 \\
\hline \multicolumn{6}{|l|}{ Health and depressive symptoms } \\
\hline Hospitalized for HF in past 2 years, \% & 51 & 41 & 0.8 & 1 & .3 \\
\hline Taking medication for HF, \%* & 87 & 86 & & & .7 \\
\hline Wave 1 Revised CES-D, mean \pm SD (range $0-8$ ) & $2.1 \pm 2.1$ & $3.8 \pm 2.6$ & -3.6 & 190 & .001 \\
\hline History of stroke, \%* & 17 & 32 & & & .1 \\
\hline \multicolumn{6}{|l|}{ Functional impairment, mean $\pm S D$} \\
\hline Total cognitive score (range 0-35) & $19.1 \pm 6.0$ & $18.7 \pm 5.3$ & 0.25 & 197 & .8 \\
\hline Mental status (range $0-10$ ) & $8.8 \pm 1.8$ & $8.8 \pm 1.9$ & 0.05 & 197 & .96 \\
\hline Immediate recall (range $0-10$ ) & $4.4 \pm 1.8$ & $4.4 \pm 1.4$ & -0.10 & 197 & .92 \\
\hline Delayed recall (range $0-10$ ) & $3.0 \pm 2.1$ & $3.0 \pm 2.0$ & -0.04 & 197 & .97 \\
\hline Serial 7s (range 0-5) & $2.9 \pm 1.8$ & $2.5 \pm 2.1$ & 0.9 & 197 & .4 \\
\hline Physical function impairment, \%* & & & & & .7 \\
\hline No impairment & 4 & 0 & & & \\
\hline Activities of daily living* & 12 & 5 & & & .5 \\
\hline Moderate mobility and strength & 31 & 36 & 0.2 & 1 & .6 \\
\hline Greater mobility and strength & 53 & 59 & 0.3 & 1 & .6 \\
\hline Any instrumental activities of daily living & 52 & 68 & 2.1 & 1 & .15 \\
\hline
\end{tabular}

${ }^{*}$ Fisher exact test used because of expected cell counts of less than 5. Sample size varied for some analyses because of missing data for one or both variables. $\mathrm{SD}=$ standard deviation; HF = heart failure; CES-D = Center for Epidemiologic Studies-Depression Scale.

pressed heart failure patients were not more cognitively impaired on the total cognitive score or any of the subscales constituting the total score. The depressed heart failure group did not report significantly greater impairment in physical function.

\section{DISCUSSION}

This study provides evidence from a community-based sample that heart failure confers a greater risk for syndromal depression than do other heart conditions. Approximately one in 10 people with heart failure in the community suffers from syndromal depression. The $11 \%$ rate of syndromal depression in this sample is lower than the $15 \%$ found by Maricle et al. ${ }^{7}$ and the $36.5 \%$ found by Koenig; ${ }^{6}$ these authors examined hospital samples. Nonetheless, the odds of heart failure patients developing depression was almost twice that of patients with other or no heart conditions, even after controlling for number of common chronic illnesses, symptomatic fatigue and apnea, and cognitive and physical impairment. Controlling for fatigue is a stringent and conservative test of the association between depression and heart failure, because fatigue is one of the more common symptoms of depression. There- fore, the increased odds of depression in the heart failure group may be an underestimate.

There was an association between depressive symptoms on the revised CES-D and heart failure, but the association was no longer significant after controlling for medical covariates and prior depressive symptoms. The report of depressive symptoms differs from a categorical diagnosis of depression, yet the exact nature of this difference needs further elaboration. In the comparison between these two multivariate analyses, it appears that medical morbidity influences this measure more than a DSM-based definition of depression. This is in keeping with prior research, in which the CES-D tends to designate a broader group of individuals as "affected." 23,24 However, this study was mostly cross-sectional and was therefore unable to definitively determine the nature of the relationship between depressive symptoms and congestive heart failure the way a longitudinal study might.

The comparison between depressed and not depressed patients with heart failure yielded few clues as to correlates of depression in this group. The sample size was considerably smaller than that for the total sample, so differences on variables such as functional impairment failed to reach sig- 
nificance. Nonetheless, prior depressive symptoms remained a strong predictor of syndromal depression.

There are several limitations of this study that must be kept in mind before interpreting the results. The logistics of assessing 6,000 people limited the quality of assessment. Lay interviewers administered psychiatric and cognitive measures. Specifically, the DSM-IV criterion for impairment related to depression was not assessed, so respondents might not have suffered syndromal depression that would be considered clinically significant. Health variables and functional impairment were based on selfreport, and confirmation by a physician or medical records was not sought. Therefore, the degree of misclassification is likely to be greater than that found in clinical studies.

This study shows that heart failure is associated with greater rates of syndromal depression in a community sample. One recent study by Murberg et al. ${ }^{11}$ found that depressed patients with heart failure had greater mortality at 2-year follow-up. Therefore, a better understanding of the nature and correlates of depression in this sample is essential for the medical and emotional management of this illness.

\section{REFERENCES}

1. Funk M, Krumholz H. Epidemiologic and economic impact of advanced heart failure. J Cardiovasc Nurs 1996;10:1-10.

2. Massie B, Shah N. Evolving trends in the epidemiologic factors of heart failure: Rationale for preventive strategies and comprehensive disease management. Am Heart J 1997;133:703-712.

3. Eriksson H, Svardsudd K, Larsson B et al. Quality of life in early heart failure. Scand J Prim Health Care 1988;6:161-167.

4. Dracup K, Walden J, Stevenson L et al. Quality of life in patients with advanced heart failure. J Heart Lung Transplant 1992;11:273-279.

5. Freedland K, Carney R, Rich M et al. Depression in elderly patients with congestive heart failure. J Geriatr Psychiatry 1991;24:59-71.

6. Koenig H. Depression in hospitalized older patients with congestive heart failure. Gen Hospital Psychiatry 1998;11:418-424.

7. Maricle R, Hosenpud J, Normal D et al. Depression in patients being evaluated for heart transplantation. Gen Hospital Psychiatry 1989;11:418-424.
8. Murberg T, Bru E, Aarsland T et al. Social support, social disability and their role as predictors of depression among patients with congestive heart failure. Scand J Soc Med 1998;2:87-95.

9. Rideout E, Montemuro M. Hope, morale and adaptation in patients with chronic heart failure. J Adv Nurs 1986;11:429-438.

10. Hawthorne M, Hixon M. Functional status, mood disturbance and quality of life in patients with heart failure. Prog Cardiovasc Nurs 1994;9:22-32.

11. Murberg T, Bru E, Svebak S et al. Depressed mood and subjective health symptoms as predictors of mortality in patients with congestive heart failure: A two-years follow-up study. Int J Psychiatry Med 1999;29:311-326.

12. Murberg T, Bru E, Aarsland T et al. Functional status and depression among men and women with congestive heart failure. Int J Psychiatry Med 1998;28: 273-291.

13. Chermerinski E, Robinson R. The neuropsychiatry of stroke. Psychosomatics 2000;41:5-14.

14. Cohen-Cole S, Stoudemire A. Major depression and physical illness: Special considerations in diagnosis and biologic treatment. Psychiatr Clin North Am 1987;10:1-17.

15. Koenig H, George L, Peterson B et al. Depression in medically ill, hospitalized, older adults. Prevelence correlates, and course of symptoms based on six diagnostic schemes. Am J Psychiatry 1997;154:1376-1383.

16. Soldo B, Hurd M, Rodgers W et al. Asset and health dynamics among the oldest old: An overview of the AHEAD study. J Gerontol B Psychol Sci Soc Sci 1997;52B:1-19.

17. Burkhauser R, Gertler P. The Health and Retirement Study: Data quality and early results. J Hum Resour 1995;30:S1-S6.

18. Kessler R, McGonagle K, Sanyang Z et al. Lifetime and 12-month prevalence of DSM-IIIR psychiatric disorders in the United States: Results from the National Comorbidity Study. Arch Gen Psychiatry 1994;51:8-19.

19. Radloff L, Teri L. Use of the Center for Epidemiologic Studies-Depression Scale with older adults. Clin Gerontologist 1986;5:119-135

20. Brandt J, Spencer M, Folstein M. The telephone interview for cognitive status. Neuropsychiatry Neuropsychol Behav Neurol 1988;1:111-117.

21. Folstein M, Folstein S, McHugh P. "Mini-mental state". A practical method for grading the cognitive state of patients for the clinician. J Psychiatry Res 1975;12:189-198.

22. Guralnik J, Ferrucci L, Simonsick E et al. Performance measures of lower extremity function in older people as predictors of subsequent disability. $\mathrm{N}$ Engl J Med 1995;332:556-561.

23. Turvey C, Wallace R, Herzog R. The relation between a revised CES-D measure of depressive symptoms and a DSM-based measure of major depressive episodes in the elderly. Int Psychogeriatr 1999;11:139-148.

24. Myers J, Weissman M. Use of a self-report symptom scale to detect the depressive syndrome. Am J Psychiatry 1980;137:1081-1084. 